\title{
A Multicenter Evaluation of Vancomycin-Associated Acute Kidney Injury in Hospitalized Patients with Acute Bacterial Skin and Skin Structure Infections
}

\author{
Sarah C. J. Jorgensen • Kyle P. Murray • Abdalhamid M. Lagnf • Sarah Melvin • Sahil Bhatia • \\ Muhammad-Daniayl Shamim · Jordan R. Smith · Karrine D. Brade · Samuel P. Simon • \\ Jerod Nagel · Karen S. Williams · Jessica K. Ortwine · Michael P. Veve · James Truong • \\ David B. Huang · Susan L. Davis · Michael J. Rybak
}

Received: November 16, 2019 / Published online: January 25, 2020

(C) The Author(s) 2020

\section{ABSTRACT}

Background: We sought to determine the realworld incidence of and risk factors for vancomycin-associated acute kidney injury (V-AKI) in hospitalized adults with acute bacterial skin and skin structure infections (ABSSSI).

Methods: Retrospective, observational, cohort study at ten U.S. medical centers between 2015

Enhanced Digital Features To view enhanced digital features for this article go to https://doi.org/10.6084/ m9.figshare.11409432.

Electronic supplementary material The online version of this article (https://doi.org/10.1007/s40121019-00278-1) contains supplementary material, which is available to authorized users.

S. C. J. Jorgensen · A. M. Lagnf · S. Melvin · S. Bhatia M.-D. Shamim · S. L. Davis · M. J. Rybak ( $ه)$ Eugene Applebaum College of Pharmacy \& Health Sciences, Wayne State University, Detroit, MI, USA e-mail:m.rybak@wayne.edu

K. P. Murray · M. J. Rybak

Detroit Medical Center, Detroit, MI, USA

J. R. Smith

Fred Wilson School of Pharmacy, High Point

University, High Point, NC, USA

J. R. Smith

Cone Health, Greensboro, NC, USA

K. D. Brade

Boston Medical Center, Boston, MA, USA and 2019. Hospitalized patients treated with vancomycin $(\geq 72 \mathrm{~h})$ for ABSSSI and $\geq$ one baseline AKI risk factor were eligible. Patients with end-stage kidney disease, on renal replacement therapy or AKI at baseline, were excluded. The primary outcome was V-AKI by the vancomycin guidelines criteria.

Results: In total, 415 patients were included. V-AKI occurred in 39 (9.4\%) patients. Independent risk factors for V-AKI were: chronic alcohol abuse (aOR 4.710, 95\% CI 1.929-11.499), no medical insurance (aOR 3.451, 95\% CI 1.310-9.090), ICU residence (aOR 4.398, 95\% CI 1.676-11.541), Gram-negative coverage (aOR 2.926, 95\% CI 1.158-7.392) and vancomycin duration (aOR 1.143, 95\% CI 1.037-1.260). Based on infection severity and comorbidities,

\section{S. P. Simon}

Maimonides Medical Center, Brooklyn, NY, USA

J. Nagel

University of Michigan, Ann Arbor, MI, USA

K. S. Williams

Guthrie Robert Packer Hospital, Sayre, PA, USA

J. K. Ortwine

Parkland Memorial Hospital, Dallas, TX, USA

M. P. Veve

College of Pharmacy, University of Tennessee

Health Sciences Center, Knoxville, TN, USA 
$34.7 \%$ of patients were candidates for oral antibiotics at baseline and $39.3 \%$ had non-purulent cellulitis which could have been more appropriately treated with a beta-lactam. Patients with V-AKI had significantly longer hospital lengths of stay (9 vs. 6 days, $p=0.001$ ), higher 30 -day readmission rates (30.8 vs. $9.0 \%$, $p<0.001)$ and increased all-cause 30-day mortality (5.1 vs. $0.3 \%, p=0.024$ )

Conclusions: V-AKI occurred in approximately one in ten ABSSSI patients and may be largely prevented by preferential use of oral antibiotics whenever possible, using beta-lactams for nonpurulent cellulitis and limiting durations of vancomycin therapy.

Keywords: Acute bacterial skin and soft tissue infection; Acute kidney injury; Nephrotoxicity; Vancomycin

\section{Key Summary Points}

In patients with at least one baseline risk factor for acute kidney injury (AKI) treated with vancomycin for acute bacterial skin and skin structure infections, vancomycin-associated acute kidney injury (V-AKI) occurred in $9.4 \%$.

Each additional day of vancomycin therapy beyond day 3 increased the odds of V-AKI by $14.3 \%$.

\footnotetext{
M. P. Veve

University of Tennessee Medical Center, Knoxville, TN, USA

J. Truong

The Brooklyn Hospital Center, Brooklyn, NY, USA

D. B. Huang

Motif BioSciences, Princeton, NJ, USA

D. B. Huang

Rutgers New Jersey Medical School, Trenton, NJ, USA
}

S. L. Davis

Henry Ford Health-System, Detroit, MI, USA

M. J. Rybak

School of Medicine, Wayne State University, Detroit, MI, USA
The majority of patients in this study could have been more appropriately treated with safer alternatives.

V-AKI may be largely prevented by preferential use of oral antibiotics whenever possible, using beta-lactams for non-purulent cellulitis and limiting durations of vancomycin therapy.

\section{INTRODUCTION}

Acute bacterial skin and skin structure infections (ABSSSIs) are the most common infections encountered in both the ambulatory and inpatient settings [1-4]. In 2013, approximately 3.4 million individuals sought emergency department care for ABSSSI in the United States [1]. Although rates have recently stabilized, this figure represents a three-fold increase over two decades earlier and has coincided with rising hospital admission rates, secondary complications and healthcare resource utilization [1, 5-7]. The increases in ABSSSI incidence and severity have been linked to the emergence, spread and persistence of community-acquired methicillin-resistant Staphylococcus aureus (CAMRSA) as a predominant pathogen [7]. Although the prevalence of invasive MRSA infections may be declining in some regions, it remains a predominant pathogen among patients with purulent ABSSSI in the US [8-11].

Vancomycin has been the standard therapy for serious ABSSSI caused by resistant Grampositive bacteria for decades $[9,12]$. It remains the antibiotic most extensively studied in ABSSSI randomized controlled clinical trials (RCTs) with efficacy and safety outcomes generally similar to new comparators [13, 14]. Guidelines recommend vancomycin for firstline treatment of ABSSSI due to suspected or confirmed MRSA with the highest strength recommendation $[9,15]$. Despite this support and its widespread use, recognition of vancomycin's shortcomings [poor tissue penetration, decreased activity against strains with higher minimum inhibitory concentrations (MICs) or the heteroresistant phenotype, need 
for therapeutic drug-monitoring and nephrotoxicity] has led many experts to question its future role in the treatment of ABSSSI $[13,16,17]$. To counter concerns about reduced effectiveness, experts have recommended more intensive vancomycin dosing in serious infections, targeting serum trough concentration levels between 15 and $20 \mathrm{mg} / \mathrm{L}$ as a surrogate for area under the concentration-time curve $(\mathrm{AUC}) \geq 400[18,19]$. More aggressive vancomycin dosing has been associated with increased reports of vancomycin-associated acute kidney injury (V-AKI) $[19,20]$. On the other hand, less intensive vancomycin dosing is recommended for non-severe ABSSSI [15], and these patients may be candidates for early transition to oral therapy, attenuating vancomycin exposure-associated renal injury. Furthermore, RCTs comparing vancomycin to other anti-MRSA agents for the treatment of ABSSSI have documented low overall rates of renal adverse events $[13,14]$. There are a number of reasons to question whether the low rates of V-AKI observed in ABSSSI RCTs can be generalized to the real-world setting. First, patients with well-described risk factors for V-AKI were either excluded or underrepresented in these studies [13]. Antibiotics are administered for a pre-specified duration, generally $7-14$ days, and in some RCTs there is the option to transition to oral therapy around day 3 [21-24]. In the realworld setting, patients have multiple risk factors, are frequently on concomitant nephrotoxic medications, and intravenous (IV) antibiotics are often continued long after patients became candidates for oral therapy $[6,19,25,26]$. This is concerning, because some studies have shown that the risk of V-AKI increases with longer durations of therapy [19]. Finally, although a clear distinction can be made between a patient presenting with necrotizing fasciitis and a stable patient with an uncomplicated abscess, the vast majority of ABSSSIs fall within a gray area between these entities, and clinicians may err on the side of caution with a more intensive vancomycin dosing schedule.

$\mathrm{V}$-AKI is typically reversible; however, AKI can further complicate a patient's hospital course and has been associated with prolonged hospital and intensive care unit (ICU) lengths of stay and increased overall mortality [19, 27-29]. Given the large number of patients treated with vancomycin for ABSSSI, V-AKI in this group would be expected to exert a tremendous burden on the healthcare system. Recent studies have demonstrated reduced rates of $\mathrm{V}$-AKI when vancomycin is dosed to target an AUC/MIC of 400-600, rather than trough-based dosing $[30,31]$. Perceived challenges with implementation of AUC-based monitoring (time allocation, training requirements, costs) may dissuade its use in non-critically ill ABSSSI patients. Newer agents with proven efficacy for MRSA infections are also available, but are not routinely prescribed for ABSSSI because of higher drug acquisition costs and/or relative lack of experience compared to vancomycin.

These considerations underscore the need to better characterize V-AKI with real-world use among hospitalized patients treated with this agent for ABSSSI. The objectives of this study were therefore to (1) determine the incidence of V-AKI with real-world use among hospitalized patients with ABSSSI, (2) identify baseline and treatment-related risk factors for $\mathrm{V}$-AKI among hospitalized ABSSSI patients, and (3) explore antimicrobial stewardship opportunities to improve appropriate vancomycin use in ABSSSIs.

\section{STUDY DESIGN}

This was a multicenter, retrospective, observational, cohort study conducted from January 2015 to June 2019 at ten US medical centers (seven academic urban and three academic rural) representative of a diverse geographic region and patient population. Approval was obtained from each center's institutional review board (IRB) with a waiver for informed consent. The Wayne State University IRB served as the coordinating IRB. Potential patients were screened from reports generated based on International Classification of Diseases, 10th Revision, Clinical Modification (ICD-10-CM) diagnostic codes for ABSSSI. Patients were eligible for the study if they met the following criteria: (1) age $\geq 18$ years; (2) IV vancomycin 
initiated $\leq 24 \mathrm{~h}$ of admission and continued for $\geq 72 \mathrm{~h}$; (3) ABSSSI per the US Food and Drug Administration definition [32]; and (4) $\geq$ one baseline risk factor for AKI (Supplementary Appendix 1) [27, 33-37]. Patients were excluded if any of the following were true: (1) baseline serum creatinine $>4.0 \mathrm{mg} / \mathrm{dL}$; (2) end-stage renal disease or receipt of chronic or emergent renal replacement therapy prior to vancomycin initiation; (3) AKI at the time of vancomycin initiation [38]; (4) concomitant infection requiring systemic antimicrobial therapy when vancomycin was initiated (patients with bacteremia thought to be from an ABSSSI source were included); or (5) pregnant or nursing mother. Relevant patient demographic, clinical, treatment and outcome data were extracted from the electronic medical record by study investigators at each center and entered into a secured electronic data collection form [39]. Patients were followed until hospital discharge. Readmissions to the same institution within 30 days of discharge were also recorded. A convenience sample of 500 patients was targeted based on feasibility and anticipated funding including 200 patients from the lead investigator's site and 30 patients each from ten additional medical centers. Due to early study termination, the target sample size was not met.

Comorbidity burden was quantified by calculating the Charlson Comorbidity score [40]. Immunosuppression was defined as previously described [41]. The systemic inflammatory response syndrome (SIRS), quick Sequential Organ Failure Assessment (qSOFA) and severe sepsis were evaluated according to consensus guidelines using the worst physiological measurements recorded within $24 \mathrm{~h}$ of vancomycin initiation [37, 42]. The Dundee classification was used to determine severity of illness at infection onset [41, 43]. The Dundee classification is a validated scoring system for ABSSSI based on routinely measured objective physiological parameters and the presence of comorbidities that may complicate or delay the resolution of infection [41, 43]. Patients in class I are candidates for oral antibiotic therapy targeted to Streptococcus pyogenes and S. aureus. Initial IV antibiotic therapy, targeted to these Gram-positive pathogens is appropriate for patients in classes II and III, while therapy should be broadened to cover Gram-negative and anaerobic bacteria for class IV $[41,43]$. Initial serum creatinine was measured within $24 \mathrm{~h}$ of the first vancomycin dose. Peak serum creatinine was the highest value recorded while receiving vancomycin to within $72 \mathrm{~h}$ of discontinuation. Glomerular filtration rate (GFR) was estimated using the Cockcroft-Gault Modification of Diet and Renal Disease (MDRD) and Chronic Kidney Disease Epidemiology Collaboration (CKD-EPI) equations [36, 44, 45] Where available, vancomycin exposure was evaluated using initial steady-state trough concentrations [19]. Vancomycin was dosed and monitored according to each hospital's standard of care.

The primary outcome was V-AKI evaluated according to vancomycin guidelines definition as an increase from baseline in serum creatinine of $\geq 0.5 \mathrm{mg} / \mathrm{L}$ or $50 \%$ on $\geq$ two consecutive measurements [18]. V-AKI was also evaluated according to the RIFLE (risk, injury, failure, loss, end-stage renal disease) and the Acute Kidney Injury Network (AKIN) criteria. [46, 47] The risk period was from vancomycin initiation to $72 \mathrm{~h}$ after the last dose or at hospital discharge, which ever occurred first [48].

\section{Statistical Analysis}

Descriptive statistics were used to characterize the cohort. Continuous variables were reported as medians (interquartile ranges, IQR), whereas categorical variables were expressed as counts and percentages. Bivariate associations between covariates and V-AKI were expressed as odds ratios and 95\% confidence intervals (CIs).

To identify independent predictors of V-AKI, we modeled both occurrence of V-AKI and time to $\mathrm{V}$-AKI. Occurrence of $\mathrm{V}$-AKI was evaluated by multivariable logistic regression. Covariates with a $p$ value less than 0.2 and with biological plausibility by unadjusted analyses were entered into the model through defined blocks starting with baseline demographic and clinical variables followed by vancomycin-related variables and other management-related variables. Stepwise techniques were used to build a parsimonious model with variables remaining in the 
Table 1 Baseline patient and hospital characteristics and unadjusted odds ratios for vancomycin-associated acute kidney injury

\begin{tabular}{|c|c|c|c|}
\hline Characteristic & $\begin{array}{l}n=415 \\
n(\%) \text { or median (IQR) }\end{array}$ & Odds ratio $(95 \%$ confidence interval $)$ & $p$ value \\
\hline Age, years & $58(46-69)$ & $0.996(0.976-1.016)$ & 0.671 \\
\hline Age $\geq 65$ years & $150(36.1)$ & $0.988(0.497-1.965)$ & 0.973 \\
\hline \multicolumn{4}{|l|}{ Sex } \\
\hline Male & $217(52.3)$ & $1.711(0.863-3.394)$ & 0.121 \\
\hline Female & $198(47.7)$ & Reference & - \\
\hline Actual body weight $(\mathrm{kg})$ & $91(74-115)$ & $1.004(0.996-1.011)$ & 0.323 \\
\hline Body mass index $\left(\mathrm{kg} / \mathrm{m}^{2}\right)$ & $31(25-39)$ & $1.010(0.986-1.034)$ & 0.416 \\
\hline Obese $\left(\mathrm{BMI} \geq 30 \mathrm{~kg} / \mathrm{m}^{2}\right)$ & $230(55.4)$ & $0.932(0.481-1.807)$ & 0.835 \\
\hline Baseline serum creatinine $(\mathrm{mg} / \mathrm{dL})$ & $0.82(0.70-1.00)$ & $1.386(0.521-3.685)$ & 0.513 \\
\hline Baseline serum creatinine $>1.5 \mathrm{mg} / \mathrm{dL}$ & $13(3.1)$ & $0.798(0.101-6.308)$ & 1.000 \\
\hline \multicolumn{4}{|l|}{ Baseline estimated GFR } \\
\hline Cockroft Gault (mL/min) & $98(71-129)$ & $1.002(0.996-1.009)$ & 0.486 \\
\hline $\mathrm{CG}<45 \mathrm{~mL} / \mathrm{min} / 1.73 \mathrm{~m}^{2}$ & $31(7.5)$ & $0.647(0.148-2.920)$ & 0.559 \\
\hline $\operatorname{MDRD}\left(\mathrm{mL} / \mathrm{min} / 1.73 \mathrm{~m}^{2}\right)$ & $94(74-114)$ & $1.000(0.990-1.009)$ & 0.943 \\
\hline $\mathrm{MDRD}<45 \mathrm{~mL} / \mathrm{min} / 1.73 \mathrm{~m}^{2}$ & $19(4.6)$ & $1.141(0.254-5.135)$ & 0.863 \\
\hline CKD-EPI $\left(\mathrm{mL} / \mathrm{min} / 1.73 \mathrm{~m}^{2}\right)$ & $97(78-117)$ & $0.998(0.986-1.009)$ & 0.687 \\
\hline CKD-EPI $<45 \mathrm{~mL} / \mathrm{min} / 1.73 \mathrm{~m}^{2}$ & $223(5.5)$ & $0.423(0.056-3.230)$ & 0.393 \\
\hline \multicolumn{4}{|l|}{ Race } \\
\hline Caucasian & $192(46.3)$ & Reference & - \\
\hline African American & $177(42.7)$ & $0.440(0.203-0.953)$ & 0.037 \\
\hline Other $^{a}$ & $46(11.1)$ & $1.102(0.412-2.885)$ & 0.843 \\
\hline \multicolumn{4}{|l|}{ Hospital } \\
\hline A & $193(46.5)$ & Reference & - \\
\hline B & $58(14)$ & $1.026(0.321-3.276)$ & 0.966 \\
\hline $\mathrm{C}$ & $30(7.2)$ & $2.769(0.910-8.428)$ & 0.073 \\
\hline $\mathrm{D}$ & $2(0.5)$ & 0 & 1.0 \\
\hline E & $30(7.2)$ & $0.477(0.060-3.789)$ & 0.484 \\
\hline $\mathrm{F}$ & $4(1.0)$ & 0 & 1.0 \\
\hline G & $30(7.2)$ & $0.989(0.212-4.618)$ & 0.989 \\
\hline $\mathrm{H}$ & $16(3.9)$ & $4.615(1.304-16.334)$ & 0.018 \\
\hline I & $22(5.3)$ & $2.186(0.572-8.361)$ & 0.253 \\
\hline
\end{tabular}


Table 1 continued

\begin{tabular}{|c|c|c|c|}
\hline Characteristic & $\begin{array}{l}n=415 n(\%) \text { or } \\
\text { median (IQR) }\end{array}$ & Odds ratio $(95 \%$ confidence interval $)$ & $p$ value \\
\hline $\mathrm{J}$ & $30(7.2)$ & $1.538(0.411-5.753)$ & 0.522 \\
\hline \multicolumn{4}{|l|}{ Medical insurance } \\
\hline Medicare & $137(33.0)$ & Reference & - \\
\hline Medicaid & $68(16.4)$ & $0.827(0.279-2.450)$ & 0.731 \\
\hline Private & $129(31.1)$ & $0.875(0.365-2.102)$ & 0.766 \\
\hline Mixed & $49(11.8)$ & $0.926(0.284-3.019)$ & 0.898 \\
\hline Uninsured & $32(7.7)$ & $3.472(1.283-9.396)$ & 0.014 \\
\hline \multicolumn{4}{|l|}{ Admission source } \\
\hline Home & $375(90.4)$ & Reference & - \\
\hline Nursing facility & $27(6.5)$ & $0.753(0.171-3.312)$ & 0.708 \\
\hline Homeless & $8(1.9)$ & $1.345(0.161-11.244)$ & 0.784 \\
\hline Other & $5(1.2)$ & 0 & 1.00 \\
\hline \multicolumn{4}{|l|}{ Social history } \\
\hline Tobacco use & $101(24.3)$ & $1.433(0.697-2.946)$ & 0.325 \\
\hline Alcohol abuse & $39(9.4)$ & $4.126(1.831-9.298)$ & $<0.001$ \\
\hline IV drug abuse & $38(9.2)$ & $1.529(0.560-4.173)$ & 0.405 \\
\hline Marijuana use & $35(8.4)$ & $0.896(0.261-3.072)$ & 0.861 \\
\hline \multicolumn{4}{|l|}{ Comorbidities } \\
\hline Heart failure & $61(14.7)$ & $1.573(0.686-3.606)$ & 0.281 \\
\hline Diabetes mellitus & $152(36.6)$ & $1.736(0.895-3.367)$ & 0.100 \\
\hline COPD & $70(16.9)$ & $1.816(0.841-3.922)$ & 0.124 \\
\hline Peripheral vascular disease & $42(10.1)$ & $1.717(0.674-4.375)$ & 0.257 \\
\hline Chronic venous insufficiency & $16(3.9)$ & $0.633(0.081-4.928)$ & 0.663 \\
\hline Cancer & $48(11.6)$ & $0.862(0.292-2.542)$ & 0.788 \\
\hline Liver disease & $24(5.8)$ & $0.404(0.053-3.075)$ & 0.366 \\
\hline Immunosuppression & $22(5.3)$ & $0.445(0.058-3.400)$ & 0.423 \\
\hline Psychiatric disorder & $65(15.7)$ & $1.714(0.772-3.804)$ & 0.181 \\
\hline Charlson Comorbidity score & $1(0-3)$ & $1.141(0.986-1.322)$ & 0.077 \\
\hline Hospitalization within 90 days & $92(22.2)$ & $1.880(0.924-3.825$ & 0.078 \\
\hline
\end{tabular}


Table 2 Baseline infection and illness severity characteristics and unadjusted odds ratios for vancomycin-associated acute kidney injury

\begin{tabular}{|c|c|c|c|}
\hline Characteristic & $\begin{array}{l}n=415 \\
n(\%) \text { or median } \\
\quad(\mathrm{IQR})\end{array}$ & $\begin{array}{l}\text { Odds ratio ( } 95 \% \text { confidence } \\
\text { interval) }\end{array}$ & $p$ value \\
\hline \multicolumn{4}{|l|}{ Dundee class } \\
\hline Class 1 & $144(34.7)$ & Reference & - \\
\hline Class 2 & $69(16.6)$ & $0.529(0.169-1.659)$ & 0.275 \\
\hline Class 3 & $136(32.8)$ & $0.909(0.416-1.988)$ & 0.811 \\
\hline Class 4 & $66(15.9)$ & $1.020(0.395-2.635)$ & 0.967 \\
\hline \multicolumn{4}{|l|}{ ABSSSI type } \\
\hline Non-purulent cellulitis & $163(39.3)$ & Reference & - \\
\hline Purulent cellulitis & $99(23.9)$ & $1.296(0.546-3.079)$ & 0.556 \\
\hline Cutaneous abscess & $92(22.2)$ & $1.099(0.438-2.758)$ & 0.841 \\
\hline Wound infection & $61(14.7)$ & $1.742(0.684-4.435)$ & 0.245 \\
\hline \multicolumn{4}{|l|}{ ABSSSI location } \\
\hline Upper extremity & $60(14.5)$ & Reference & \\
\hline Lower extremity & $248(59.8)$ & $1.100(0.432-2.796)$ & 0.842 \\
\hline Torso & $57(13.7)$ & $0.327(0.063-1.694)$ & 0.183 \\
\hline Buttock/perianal & $24(5.8)$ & $0.818(0.153-4.370)$ & 0.814 \\
\hline Head/neck & $26(6.3)$ & $0.750(0.141-3.988)$ & 0.736 \\
\hline Blood culture obtained & $293(70.6)$ & $2.014(0.864-4.697)$ & 0.099 \\
\hline Positive blood culture & $20(4.8)$ & $1.075(0.240-4.816)$ & 0.925 \\
\hline Failed outpatient therapy for index ABSSSI & $94(22.4)$ & $1.195(0.559-2.551)$ & 0.646 \\
\hline Abnormal temperature $(>38 \mathrm{C}$ or $<36 \mathrm{C})$ & $96(23.1)$ & $1.163(0.545-2.481)$ & 0.696 \\
\hline Respiratory rate $\geq 22$ breath/minute & $76(18.3)$ & $0.795(0.321-1.970)$ & 0.619 \\
\hline Heart rate $>90$ beats/minute & $262(63.1)$ & $1.350(0.663-2.750)$ & 0.407 \\
\hline Systolic blood pressure $\leq 100 \mathrm{mmHg}$ & $87(21.0)$ & $0.970(0.429-2.194)$ & 0.942 \\
\hline Abnormal WBC $\left(>12 \times 10^{3}\right.$ or $\left.<4 \times 10^{3}\right)$ & $200(48.2)$ & $0.815(0.419-1.584)$ & 0.546 \\
\hline Altered mental status $(\mathrm{GCS}<15)$ & $20(4.8)$ & $2.571(0.815-8.115)$ & 0.096 \\
\hline $\mathrm{qSOFA} \geq 2$ & $41(9.9)$ & $1.389(0.511-3.774)$ & 0.518 \\
\hline$\geq 2$ SIRS criteria present & $202(48.7)$ & $1.122(0.580-2.170)$ & 0.732 \\
\hline Severe sepsis/septic shock & $13(3.1)$ & $1.794(0.383-8.401)$ & 0.452 \\
\hline $\begin{array}{l}\text { ICU admission within } 24 \mathrm{~h} \text { of hospital } \\
\text { admission }\end{array}$ & $30(7.2)$ & $4.152(1.708-10.097)$ & 0.001 \\
\hline
\end{tabular}


Table 2 continued

\begin{tabular}{|c|c|c|c|}
\hline Characteristic & $\begin{array}{l}n=415 n(\%) \text { or } \\
\text { median (IQR) }\end{array}$ & $\begin{array}{l}\text { Odds ratio ( } 95 \% \text { confidence } \\
\text { interval) }\end{array}$ & $p$ value \\
\hline $\begin{array}{l}\text { Mechanical ventilation within } 24 \mathrm{~h} \text { of } \\
\text { admission }\end{array}$ & $7(1.7)$ & $4.011(0.752-21.397)$ & 0.080 \\
\hline Skin/tissue/fluid specimen obtained ${ }^{b}$ & $195(77.4)$ & $0.348(0.150-0.809)$ & 0.014 \\
\hline Staphylococcus aureus ${ }^{\mathrm{b}}$ & $102(40.5)$ & & \\
\hline MSSA $^{b}$ & $33(13.1)$ & & \\
\hline $\mathrm{MRSA}^{\mathrm{b}}$ & $69(27.4)$ & & \\
\hline Streptococcus spp. ${ }^{\mathrm{b}}$ & $35(13.9)$ & & \\
\hline Gram-negative $^{\mathrm{b}}$ & $30(11.9)$ & & \\
\hline Escherichia coli & $7(2.8)$ & & \\
\hline Klebsiella pneumonia & $6(2.4)$ & & \\
\hline Pseudomonas aeruginosa & $4(1.6)$ & & \\
\hline No organisms/normal flora ${ }^{b, c}$ & $41(16.3)$ & & \\
\hline
\end{tabular}

ABSSSI acute bacterial skin and skin structure infection, $B M I$ body mass index, $C$ Celsius, $C G$ Cockroft-Gault, $C K D-E P I$ chronic kidney disease epidemiology collaboration, $C T$ computed tomography, GCS Glasgow Coma Scale, $I C U$ intensive care unit, MDRD Modification of Diet in Renal Disease, MSSA methicillin-sensitive S. aureus, MRSA methicillin-resistant $S$. aureus, $M R I$ magnetic resonance imaging, $O R$ operating room, SIRS systemic inflammatory response syndrome, $q S O F A$ quick Sequential Organ Failure Assessment, $W B C$ white blood cell count

${ }^{a}$ Latino $\mathrm{n}=20$, Asian $\mathrm{n}=3$, Unknown $n=23$

${ }^{\mathrm{b}} \mathrm{n}=252$ excluding patients with non-purulent cellulitis from whom a culture could not be obtained

c Normal flora as per local laboratory definitions

final model if the adjusted $p$ value was $<0.05$. Multi-collinearity was assessed via the variance inflation factor, with values $<3$ being considered acceptable. Effect modification was also evaluated. To examine time to V-AKI, we employed Cox proportional hazards regression. Patients who did not experience V-AKI were censored $72 \mathrm{~h}$ after the last dose of vancomycin or at hospital discharge, which ever occurred first.

Two sensitivity analyses were performed: (1) excluding cases enrolled from the Detroit Medical Center which was our largest contributor, and (2) excluding sites that contributed less than ten cases.

All calculations were performed with SAS 9.4 Statistical Software (SAS Institute, Cary, NC, USA) and SPSS, v.25 (IBM. Armonk, NY, USA).

\section{RESULTS}

A total of 415 patients met inclusion criteria and were evaluated, representing 57,143 vancomycin-days. Demographic, clinical and infection characteristics are shown in Tables 1 and 2 . The median age was 58 (46-69) years and approximately half $(52.3 \%)$ were male. Both Caucasian and African American patients were well represented $(46.3 \%$ and $42.7 \%$, respectively). The majority of patients $(55.4 \%)$ were obese. Diabetes mellitus (36.6\%), chronic obstructive pulmonary disease $(16.9 \%)$ and psychiatric illnesses (15.7\%) were the most common comorbidities. The median GFR at initial evaluation ranged from $94(71-129) \mathrm{mL} /$ $\mathrm{min} / 1.73 \mathrm{~m}^{2}$ (MDRD) to 98 (71-129) $\mathrm{mL} / \mathrm{min}$ (Cockroft-Gault) with few patients (4.6-7.5\%) 
having a GFR under $45 \mathrm{~mL} / \mathrm{min}$. Nearly half $(48.7 \%)$ of patients meet criteria for sepsis but few patients $(3.1 \%)$ had severe sepsis at initial evaluation. With regards to the Dundee classification for infection severity, 144 (34.7\%) were in severity class I, $69(16.6 \%)$ in class II, 136 $(32.8 \%)$ in class III and $66(15.9 \%)$ in class IV. The most common ABSSSI type and location were non-purulent cellulitis (39.3\%) and lower extremity (59.8\%), respectively.

ABSSSI management is summarized in Table 3. Among patients from whom a tissue or fluid specimen could potentially be obtained (i.e., purulent cellulitis, abscess and wound infection, $n=252$ ), a culture was collected in $77.4 \%$. S. aureus was the most frequently cultured bacterial species (MSSA 13.1\% and MRSA $27.4 \%)$. Twelve percent of cultures were positive for Gram-negative bacteria. Overall, blood cultures were collected from $70.6 \%$ of patients. Blood cultures were obtained more frequently as Dundee class increased from I to IV $(59.7 \%$, $55.1 \%, 78.7 \%, 93.9 \% ; p<0.001$ for trend). Approximately $5 \%$ of the cohort had secondary bacteremia (MSSA $1.2 \%$ and MRSA 2.9\%). Infectious disease services were consulted in $57.8 \%$ of cases, radiological testing was performed in $57.8 \%$ and a source control procedure was performed in $41.9 \%$. No interactions between these variables and Dundee class were observed.

With regards to vancomycin therapy, the median daily dose was $26(17-31) \mathrm{mg} / \mathrm{kg}$. Daily doses $>4000 \mathrm{mg}$ were uncommon $(4.1 \%)$ and loading doses were used in $11.3 \%$. The median duration of vancomycin was 5 (4-6) days. Vancomycin was administered for greater than 7 days to $20.7 \%$ of patients. In the subgroup of patients that had a skin specimen culture collected $(n=195)$, the length of vancomycin therapy [5 (4-6) vs. 5 (4-7), $p=0.888$ ] and the proportion of patients that received $>7$ days of vancomycin $(18.8 \%$ vs $20.6 \%, p=0.765)$, was similar in patients with and without an MRSA positive culture, respectively.

In total, $257(61.9 \%)$ patients had at least one steady-state vancomycin trough measured; the median initial trough was 12.0 (8.4-16.0) $\mathrm{mg} / \mathrm{L}$ and $68.5 \%$ of patients had an initial trough $<15 \mathrm{mg} / \mathrm{L}$. Concomitant antibiotics with Gram-negative activity and/or antipseudomonal activity were used for $\geq 72 \mathrm{~h}$ in $59.8 \%$ and $42.9 \%$ of patients, respectively. The use of concomitant nephrotoxic medicines was also common $(61.9 \%)$ and $23.4 \%$ of patients received parenteral contrast dye.

V-AKI according to the vancomycin consensus guidelines, the RIFLE and AKIN criteria, developed in $39(9.4 \%), 50(12.0 \%)$ and 64 $(15.4 \%)$ patients, respectively (Table 4$)$. The median time to V-AKI onset was 4 (3-6) days. Amongst patients who experienced V-AKI, vancomycin was discontinued due to $\mathrm{V}$-AKI in $26(61.5 \%)$. V-AKI resolution occurred before or at the time of discharge in $26(61.5 \%)$ patients. Resolution was not associated with vancomycin discontinuation; resolution occurred in $65.4 \%$ who continued vancomycin compared to $53.8 \%$ who discontinued vancomycin due to V-AKI $(p=0.485)$. Nephrology was consulted in 13 (3.1\%) patients with V-AKI and emergent hemodialysis was initiated in 4 (1.0\%).

Bivariate associations between baseline demographic, clinical and infection variables and V-AKI are shown in Tables 1 and 2. No medical insurance, chronic alcohol abuse and ICU admission within $24 \mathrm{~h}$ of vancomycin initiation were positively associated with V-AKI, while African American race was protective $(p<0.05)$. Notably, in our cohort, there was no significant association between V-AKI and age, obesity, or baseline GFR. With regards to vancomycin-related variables (Table 3 ), receipt of higher doses, including doses $>4000 \mathrm{mg} /$ day and loading doses, were not associated with an increased odds of V-AKI. Measurement of at least one vancomycin trough level was associated with an increased risk of V-AKI $(p=0.043)$. Although the number of patients with a trough $>20 \mathrm{mg} / \mathrm{L}$ was small $(n=25)$, supratherapeutic troughs were associated with increased V-AKI (OR 4.035, 95\% CI 1.297-12.555). Longer durations of vancomycin therapy were associated with increased risk particularly among the approximately $20 \%$ of patients who received vancomycin for $>7$ days (OR 2.364, 95\% CI 1.171-4.775). The receipt of parenteral contrast dye or at least one concomitant nephrotoxic medication for $\geq 48 \mathrm{~h}$ was associated with a greater than two-fold 
Table 3 Management-related variables and unadjusted odds ratios for vancomycin-associated acute kidney injury

\begin{tabular}{|c|c|c|c|}
\hline & $\begin{array}{l}n=415 \\
n(\%) \text { or median } \\
(\mathrm{IQR})\end{array}$ & $\begin{array}{l}\text { Odds ratio ( } 95 \% \text { confidence } \\
\text { interval) }\end{array}$ & $P$ value \\
\hline Radiological test performed & $240(57.8)$ & $1.514(0.755-3.038)$ & 0.240 \\
\hline $\mathrm{X}$-ray & $100(24.1)$ & & \\
\hline CT-scan & $101(24.3)$ & & \\
\hline Ultrasound & $66(15.9)$ & & \\
\hline MRI & $34(8.2)$ & & \\
\hline Surgical consult & $255(61.4)$ & $1.461(0.718-2.973)$ & 0.294 \\
\hline Dermatology consult & $8(1.9)$ & $0.979(0.964-0.993)$ & 1.000 \\
\hline Infectious diseases consult & $240(57.8)$ & $1.514(0.755-3.038)$ & 0.240 \\
\hline Source control procedure & $174(41.9)$ & $0.960(0.491-1.876)$ & 0.905 \\
\hline Bedside incision and drainage & $62(14.9)$ & & \\
\hline OR incision and drainage & $100(24.1)$ & & \\
\hline Debridement & $10(2.4)$ & & \\
\hline Amputation & $5(1.2)$ & & \\
\hline $\begin{array}{l}\text { Concomitant antipseudomonal } \\
\text { antibiotic } \geq 72 \mathrm{~h}\end{array}$ & $178(42.9)$ & $2.603(1.311-5.168)$ & 0.006 \\
\hline Cefepime & $84(20.2)$ & $1.407(0.657-3.016)$ & 0.380 \\
\hline Piperacillin-tazobactam & $83(20.0)$ & $2.831(1.411-5.681)$ & 0.003 \\
\hline Receipt of IV contrast dye & $97(23.4)$ & $2.241(1.124-4.468)$ & 0.019 \\
\hline Concomitant nephrotoxic drug $\geq 48 \mathrm{~h}$ & $257(61.9)$ & $2.572(1.151-5.748)$ & 0.021 \\
\hline Piperacillin-tazobactam & $86(20.7)$ & $2.685(1.340-5.379)$ & 0.005 \\
\hline Vasopressors & $2(0.5)$ & $9.868(0.605-160.960)$ & 0.179 \\
\hline Loop diuretic & $81(19.5)$ & $1.977(0.954-4.097)$ & 0.063 \\
\hline ACE-inhibitor & $64(15.4)$ & $1.224(0.515-2.908)$ & 0.646 \\
\hline Angiotensin receptor blocker & $25(6.0)$ & $0.830(0.188-3.659)$ & 1.000 \\
\hline Tenofovir disoproxil fumarate & $4(1.0)$ & $3.272(0.332-32.234)$ & 0.327 \\
\hline Non-steroidal anti-inflammatory & $79(19.0)$ & $0.600(0.227-1.587)$ & 0.299 \\
\hline Acyclovir & $5(1.2)$ & $6.721(1.088-41.513)$ & 0.018 \\
\hline
\end{tabular}

$A C E$ angiotensin converting enzyme, $I V$ intravenous; operating room

${ }^{a} n=257$ 
Table 4 Vancomycin-associated acute kidney injury

\begin{tabular}{ll}
\hline Definition & $\begin{array}{l}n=4 \mathbf{1 5} \\
\boldsymbol{n}(\%)\end{array}$ \\
\hline Consensus Guidelines definition & $39(9.4)$ \\
RIFLE & $50(12.0)$ \\
Risk & $32(7.7)$ \\
Injury & $10(2.4)$ \\
Failure & $8(1.9)$ \\
AKIN & $64(15.4)$ \\
Stage 1 & $47(11.3)$ \\
Stage 2 & $10(2.4)$ \\
Stage 3 & $7(1.7)$ \\
\hline
\end{tabular}

AKIN acute kidney injury network, RIFLE risk, injury, failure, loss of function, end-stage renal diseases

increased odds of V-AKI (Table 3). The use of concomitant Gram-negative coverage ( $\geq 72 \mathrm{~h}$ ) was associated with an even higher odds of V-AKI (OR 4.119, 95\% CI 1.685-10.065).

Baseline variables independently associated with the occurrence of V-AKI in the multivariable logistic regression analysis were (Table 5): no medical insurance (aOR 3.451, 95\% 1.310-9.090), chronic alcohol abuse (aOR 4.710, 95\% CI 1.929-11.499) and ICU admission within the first $24 \mathrm{~h}$ of vancomycin therapy (aOR 4.398, 95\% 1.676-11.541). After controlling for these variables, receipt of Gramnegative coverage (aOR 2.926, 95\% CI 1.158-7.392) and vancomycin duration (aOR $1.143,95 \% 1.037-1.260)$ were positive predictors of V-AKI.

The Cox proportional hazard model identified the following baseline variables significantly associated with V-AKI (Table 6): no medical insurance (aHR 2.898, 95\% CI 1.303-6.445), chronic alcohol abuse (aHR 3.295 , 95\% CI 1.559-6.964), chronic pulmonary disease (aHR 2.151, 95\% CI 1.027-4.503) and ICU admission within $24 \mathrm{~h}$ of vancomycin initiation (aHR 3.327, 95\% CI 1.487-7.446). After controlling for these variables, the only treatment-related variable independently associated with V-AKI was receipt of concomitant Gramnegative coverage (aHR 2.812, 95\% CI 1.160-6.817).

We performed exploratory analyses to better understand the relationship between chronic alcohol abuse, no medical insurance and V-AKI. First, in patients with chronic alcohol abuse, the presence of most comorbid conditions was not higher with the exception of chronic liver disease ( 4.5 vs. $17.9 \% ; p=0.001)$ but there was no interaction between liver disease and alcohol abuse for V-AKI. Baseline serum creatinine, GFR and the presence of sepsis were similar. We did not discover differences in vancomycin dosing nor was there evidence of differential vancomycin exposure based on trough levels or

Table 5 Final logistic regression model for the occurrence of vancomycin-associated acute kidney injury

\begin{tabular}{lll}
\hline Parameter & $\begin{array}{l}\text { Adjusted odds ratio } \\
(\mathbf{9 5 \%} \text { confidence interval) }\end{array}$ & $\boldsymbol{p}$ value \\
\hline No medical insurance & $3.451(1.310-9.090)$ & 0.012 \\
Chronic alcohol abuse & $4.710(1.929-11.499)$ & 0.001 \\
ICU admission within 24h of vancomycin initiation & $4.398(1.676-11.541)$ & 0.003 \\
Receipt of Gram-negative coverage $^{2}$ & $2.926(1.158-7.392)$ & 0.023 \\
Vancomycin duration $^{\mathrm{a}}$ & $1.143(1.037-1.260)$ & 0.007 \\
\hline
\end{tabular}

$A U C$ area under the curve, $I C U$ intensive care unit

Overall $p$ value (likelihood ration test) was $<0.001$; Hosmer-Lemeshow test $p=0.340$; AUC 0.776 (95\% CI $0.700-0.851$ )

${ }^{a}$ The adjusted odds ratio for vancomycin duration reflects the increased likelihood of V-AKI for each one day increase in vancomycin length of therapy 
Table 6 Final Cox proportional hazards model for time to occurrence of vancomycin-associated acute kidney injury

\begin{tabular}{llc}
\hline Parameter & Adjusted hazard ratio $\mathbf{9 5 \%}$ confidence interval) & $\boldsymbol{p}$ value \\
\hline No medical insurance & $2.898(1.303-6.445)$ & 0.009 \\
Chronic alcohol abuse & $3.295(1.559-6.964)$ & 0.002 \\
Chronic pulmonary disease & $2.151(1.027-4.503)$ & 0.042 \\
ICU admission within 24h of vancomycin initiation & $3.327(1.487-7.446)$ & 0.003 \\
Receipt of Gram-negative coverage & $2.812(1.160-6.817)$ & 0.022 \\
\hline
\end{tabular}

$I C U$ intensive care unit

The final $\log$ likelihood ratio was-202.24. All covariates were dichotomized. The overall $p$ value was $<0.001$

vancomycin duration. We did however find that blood cultures were collected more often in these patients (68.9 vs. $87.2 \% ; p=0.017)$ and concomitant piperacillin-tazobactam was given to a higher proportion of patients with chronic alcohol abuse (18.4 vs. $35.9 \% ; p=0.009)$. Chronic alcohol abuse and concomitant piperacillin-tazobactam appeared to act synergistically to increase V-AKI risk: among patients who did not receive piperacillin-tazobactam, patients with chronic alcohol abuse was associated with an increased odds of V-AKI, although the association was not statistically significant (OR 2.733, 95\% CI 0.856-8.731) but the association of chronic alcohol abuse and $\mathrm{V}$-AKI was more pronounced in those who also received piperacillin-tazobactam (OR 5.00, 95\% CI 1.405-17.793). Gram-negative bacteria were isolated in a similar proportion of patients without and with chronic alcohol abuse (15.9 vs. $10.5 \% ; p=0.743)$. Next, with regards to uninsured patients, when compared to patients with some form of medical insurance, uninsured patients were significantly younger [59 (47-70) years vs. 51 (39-60) year;, $p=0.027$ ], had higher GFR by the Cockroft-Gault equation [95 (68-129) $\mathrm{mL} / \mathrm{min}$ vs. 118 (89-154) $\mathrm{mL} / \mathrm{min}$; $p=0.016]$ and achieved lower vancomycin trough levels [12 (9-17) mg/L vs. 8 (6-10) mg/L; $p<0.001]$. Conversely, uninsured patients were more likely to receive a concomitant nephrotoxic drug (59.5 vs $78.1 \% ; p=0.038$ ), in particular piperacillin-tazobactam (16.4 vs. $62.5 \% ; p<0.001)$. However, the association between no medical insurance and V-AKI was qualitatively similar in those who did and did not receive a concomitant nephrotoxic drug (OR 3.466, 95\% CI 1.309-9.177 and OR 3.063, 95\% CI 0.328-28.577, respectively).

As shown in Table 1, rates of $\mathrm{V}$-AKI varied widely across centers. Sensitivity analyses demonstrated that the final multivariable logistic regression model had similar discrimination and satisfactory calibration when patients enrolled from the Detroit Medical Center $(n=193)$ were excluded as well as when patients enrolled from sites that contributed less than ten cases were excluded (Supplementary Appendix 2).

With regards to other important patient outcomes, length of stay was significantly longer in patients who experienced V-AKI [9 (6-3) days vs. 6 (5-9) days; $p=0.001]$ and a significantly higher proportion of patients who experienced V-AKI were readmitted to the hospital within 30 days of discharge (30.8 vs. 9.0\%; $p<0.001)$. Thirty-day all-cause mortality occurred in three patients: one $(0.3 \%)$ in the non V-AKI group compared to two $(5.1 \%)$ in the V-AKI group $(p=0.024)$.

\section{DISCUSSION}

Vancomycin is the most commonly prescribed antibiotic in US hospitals with recent studies finding approximately one in four patients receive vancomycin during the course of their hospital stay [49]. Among pharmacists, nephrologists and infectious diseases physicians, there is a broad range of beliefs about vancomycin's propensity to cause V-AKI [50]. Previous studies have reported V-AKI rates 
ranging from less than 5\% to greater than $40 \%$ and a wide variety of risk factors have been implicated [19, 33]. We conducted a focused analysis of this adverse effect specifically in hospitalized patients treated with vancomycin for ABSSSI. Our study sample was enriched with patients at higher risk of V-AKI because these patients have been poorly represented in recent randomized controlled ABSSSI studies. We believe our cohort is more broadly representative of real-world ABSSSI patients managed in the inpatient setting. We found that nearly one in ten patients experienced V-AKI. This represents a two- to three-fold increase over recent studies conducted for regulatory approval of new antibiotics that used vancomycin as the comparator and enrolled patients at low baseline risk [22-24]. Consistent with previous studies $[19,27]$, we found that patients who experienced V-AKI had significantly longer hospital lengths of stay, higher 30-day readmission rates and higher all-cause 30-day mortality. Although we are cautious not to ascribe causation, it is clear that patients who experience V-AKI place a significant burden on the healthcare system. In our study, the median length of stay of those who experienced V-AKI was 3 days longer than those who did not, which could translate into incremental costs to the hospital for room and board alone of approximately US\$8000 depending on the geographic location and type of institution [51]. Additional costs are incurred for pharmacist's time, laboratory monitoring, nephrology consult (required in $3.1 \%$ of our cohort) and dialysis $(1.0 \%$ of our cohort). These findings underscore the need to better understand the individual patient- and drug-related risk factors that would enable clinicians to safely and effectively use vancomycin when it is indicated.

Some, but not all, previous studies have found increased rates of $\mathrm{V}$-AKI with higher vancomycin trough levels $[19,27,48]$. In our cohort, higher initial troughs within the therapeutic range $(<20 \mathrm{mg} / \mathrm{mL})$ were not positively associated with V-AKI. Supratherapeutic levels dramatically increased risk; however, few patients had trough levels above $20 \mathrm{mg} / \mathrm{L}$ resulting in imprecise estimates. Vancomycin is predominantly eliminated in the urine by glomerular filtration, therefore a decrease in renal function, from any etiology, will increase vancomycin serum concentrations $[19,50]$. In addition, changes in serum creatinine are known to lag actual renal injury. This makes it difficult to establish an exposure-nephrotoxicity relationship. Aware of this, we restricted our analysis to initial trough levels which may explain the absence of an association for levels of $15-20 \mathrm{mg} / \mathrm{L}$ compared to other studies that have included levels collected throughout the course of therapy $[19,33]$.

Unlike previous studies, we also did not observe a positive relationship between vancomycin dose and V-AKI [33, 52]. However, less than $5 \%$ of patients received doses exceeding $4000 \mathrm{mg} /$ day and the median daily dose $(26 \mathrm{mg} / \mathrm{kg})$ was less than the standard $30 \mathrm{mg} /$ $\mathrm{kg} /$ day $(15 \mathrm{mg} / \mathrm{kg}$ every $12 \mathrm{~h})$. Loading doses were also uncommon (11.4\%). The majority of our patients were obese. Other studies have found obesity to be a significant risk factor for V-AKI $[18,52]$. Cognizant of this association, clinicians may have under-dosed heavier patients, minimizing our ability to detect a relationship between higher doses or obesity and V-AKI. The observation that patients who received vancomycin therapeutic drug monitoring (TDM) was associated with over a twofold increased odds of V-AKI also suggests that clinicians were more inclined to monitor vancomycin therapy in individuals perceived to be at higher risk or perhaps when longer durations of therapy were planned. The fact that TDM did not entirely obviate this risk attests to limitations of trough level monitoring and underscores the need to minimize vancomycin use when safer alternatives are suitable, particularly in patients known to be at risk for V-AKI due to concomitant nephrotoxic medications and/or other risk factors.

Prior studies have found a significant association between longer vancomycin treatment durations and risk for developing V-AKI $[18,27,53]$. We found that each additional day of therapy beyond $72 \mathrm{~h}$ was independently associated with $14 \%$ increased odds of V-AKI, and that durations greater that 7 days conferred a greater than two-fold increased odds on univariate analysis. There is now overwhelming 
data supporting short-course therapy in patients with a variety of acute bacterial infections including hospitalized patients $[54,55]$. Our study demonstrates the need to translate this data into current clinical practice to minimize iatrogenic harms of antibiotic therapy.

We found that novel risk factors, such as the absence of medical insurance and chronic alcohol abuse, were associated with V-AKI in our cohort. Liver disease was more prevalent among patients with chronic alcohol abuse and, even though baseline serum creatinine and GFR were similar in when stratified by chronic alcohol abuse and liver disease, patients with advanced liver disease may have renal impairment that is substantially more severe than suggested by serum creatinine concentration [56]. We found that concomitant nephrotoxic drugs, in particular piperacillin-tazobactam, were used more frequently in those with alcohol abuse and uninsured patients; however, this did not fully explain the increased V-AKI risk. This, coupled with the observation that blood cultures were collected more often in patients with chronic alcohol abuse, implies that the clinical picture may have suggested a more severe or complicated infection despite the fact that there was no difference in the objective signs we collected. These novel findings underscore the need to better characterize the epidemiology of ABSSSI in these vulnerable patient populations so that effective and safe management strategies can be developed.

One of the most important findings of this study was that IV vancomycin was not indicated in a large number of enrolled patients. Nearly $40 \%$ of patients had non-purulent cellulitis for which the predominant pathogen remains beta-hemolytic streptococcus, even in the post-CA-MRSA era [57, 58]. Narrow spectrum beta-lactams, which are not nephrotoxic (except for rare cases of interstitial nephritis) [59], are the drugs of choice for these infections [15]. In addition, over one-third of patients were candidates for upfront oral therapy based on meeting Dundee class I criteria. Provider perception that infections require IV antibiotics is a common reason for hospital admission in patients with ABSSSI. We now have a number of well-tolerated oral antibiotics with high oral bioavailability and appropriate spectrums of activity to treat ABSSSI [60-64]. Interventions targeted to stopping the continued overuse of parenteral antibiotics in the inpatient setting are needed.

Nearly $60 \%$ of patients in this study received combination therapy with a Gram-negative active antibiotic, yet only $16 \%$ of patients exhibited the high-risk features for which it is indicated (Dundee class IV). Exposing patients to antibiotics with an unnecessarily broadspectrum of activity is unacceptable in the current era of escalating antimicrobial resistance and the increasing incidence and severity of Clostridiodes difficile-associated diarrhea [65]. In addition, receipt of Gram-negative coverage was strongly and independently associated with V-AKI. Mounting evidence suggests that the combination of vancomycin and piperacillintazobactam is associated with increases in serum creatinine $[35,66]$. Although our sample size was not large enough to permit meaningful exploration, the risk in our study did not appear to be restricted to concomitant use of piperacillin-tazobactam only.

Our study has several important limitations. First, the study design was retrospective and observational and is therefore subject to the risk of bias inherent in this design. However, indepth chart reviews allowed us to obtain detailed patient-level data and include variables that have not been assessed before. Next, we only enrolled patients treated with vancomycin and did not include a control group. As observed in this study, patients treated with vancomycin frequently have comorbidities and baseline organ impairment and are exposed to other agents which can be harmful to the kidneys, therefore we were not able to evaluate the attributable risk from vancomycin itself. However, our study reflects real-world practice since hospitalized patients often receive concomitant nephrotoxins and have multiple comorbidities. Our study was terminated early because of the sponsor's financial status and we did not enroll our target sample size. Nearly half of the cohort were enrolled from one medical center, limiting generalizability. We observed wide ranges of V-AKI across sites with outliers often being sites that enrolled fewer patients. For these reasons, 
we conducted sensitivity analyses and found the results of multivariable analyses to be broadly consistent across sites. Antibiotic developers often fund valuable real-world research, and there is a desperate need for alternative payment models to sustain antibiotic development and related research.

\section{CONCLUSION}

In conclusion, we found that nearly $10 \%$ of hospitalized ABSSSI patients treated with vancomycin experienced V-AKI. This adverse effect exerts a tremendous burden on the patients and the healthcare system and is largely preventable with three key changes in practice: (1) use oral antibiotics whenever appropriate, (2) treat non-purulent cellulitis with beta-lactams, and (3) when vancomycin must be used for ABSSSI, limit the duration of therapy to the absolute minimum.

\section{ACKNOWLEDGEMENTS}

Funding. This study was supported by an investigator-initiated grant from Motif BioSciences Inc., Princeton, NJ, USA. No extra funding was received for the publication of this article.

Authorship. All named authors meet the International Committee of Medical Journal Editors (ICMJE) criteria for authorship for this article, take responsibility for the integrity of the work as a whole, and have given their approval for this version to be published.

Prior Presentation. This study was presented, in part, at the 29th ECCMID, Amsterdam, Netherlands, 13-16 April 2019, Poster P2297 and ID Week 2-6 October 2019, Washington DC, U.S.A. Poster 442

Disclosures. Michael J Rybak is a research support, consultant or speaker for Allergan, Melinta, Merck, Motif BioSciences, Nabriva, Paratek, Tetraphase and Shionogi. Michael J
Ryback is also the Editor-in-Chief of this journal. David B Huang is an employee of Motif BioSciences. Susan L David is a consultant for Sperow and Tetraphase. Sarah C. J. Jorgensen's current affiliation is the Mount Sinai Hospital, Toronto, Ontario, Canada. Kyle P. Murray, Abdalhamid M. Lagnf, Sarah Melvin, Sahil Bhatia, Muhammad-Daniayl Shamim, Jordan R. Smith, Karrine D. Brade, Samuel P. Simon, Jerod Nagel, Karen S. Williams, Jessica K. Ortwine, Michael P. Veve and James Truong have nothing to disclose.

Compliance with Ethics Guidelines. Approval was obtained from each center's institutional review board with a waiver for informed consent. The Wayne State University IRB served as the coordinating IRB.

Open Access. This article is licensed under a Creative Commons Attribution 4.0 International License, which permits use, sharing, adaptation, distribution and reproduction in any medium or format, as long as you give appropriate credit to the original author(s) and the source, provide a link to the Creative Commons licence, and indicate if changes were made. The images or other third party material in this article are included in the article's Creative Commons licence, unless indicated otherwise in a credit line to the material. If material is not included in the article's Creative Commons licence and your intended use is not permitted by statutory regulation or exceeds the permitted use, you will need to obtain permission directly from the copyright holder. To view a copy of this licence, visit http:// creativecommons.org/licenses/by/4.0/.

\section{REFERENCES}

1. Hersh AL, Chambers HF, Maselli JH, et al. National trends in ambulatory visits and antibiotic prescribing for skin and soft-tissue infections. Arch Intern Med. 2008;168:1585-91.

2. Jenkins TC, Sabel AL, Sarcone EE, et al. Skin and soft-tissue infections requiring hospitalization at an academic medical center: opportunities for 
antimicrobial stewardship. Clin Infect Dis. 2010;51: 895-903.

3. Klein E, Smith DL, Laxminarayan R. Hospitalizations and deaths caused by methicillin-resistant Staphylococcus aureus, United States, 1999-2005. Emerg Infect Dis. 2007;13:1840-6.

4. Edelsberg J, Taneja C, Zervos M, et al. Trends in US hospital admissions for skin and soft tissue infections. Emerg Infect Dis. 2009;15:1516-8.

5. Pollack CV Jr, Amin A, Ford WT Jr, et al. Acute bacterial skin and skin structure infections (ABSSSI): practice guidelines for management and care transitions in the emergency department and hospital. J Emerg Med. 2015;48:508-19.

6. Itani KM, Merchant S, Lin SJ, et al. Outcomes and management costs in patients hospitalized for skin and skin-structure infections. Am J Infect Control. 2011;39:42-9.

7. Tattevin P, Schwartz BS, Graber CJ, et al. Concurrent epidemics of skin and soft tissue infection and bloodstream infection due to community-associated methicillin-resistant Staphylococcus aureus. Clin Infect Dis. 2012;55:781-8.

8. Moran GJ, Krishnadasan A, Gorwitz RJ, et al. Methicillin-resistant $S$. aureus infections among patients in the emergency department. N Engl J Med. 2006;355:666-74.

9. Stevens DL, Bisno AL, Chambers HF, et al. Practice guidelines for the diagnosis and management of skin and soft tissue infections: 2014 update by the infectious diseases society of America. Clin Infect Dis. 2014;59:147-59.

10. CDC. Antibiotic resistance threats in the United States, 2019. Atlanta: US Department of Health and Human Services, CDC; 2019.

11. Acree ME, Morgan E, David MZS. S. aureus infections in Chicago, 2006-2014: increase in CA MSSA and decrease in MRSA incidence. Infect Control Hosp Epidemiol. 2017;38:1226-34.

12. Levine DP. Vancomycin: a history. Clin Infect Dis. 2006;42(Suppl 1):S5-12.

13. Savoldi A, Azzini AM, Baur D et al. Is there still a role for vancomycin in skin and soft-tissue infections? Curr Opin Infect Dis 2017.

14. Tsoulas C, Nathwani D. Review of meta-analyses of vancomycin compared with new treatments for Gram-positive skin and soft-tissue infections: are we any clearer? Int J Antimicrob Agents. 2015;46: $1-7$.
15. Liu C, Bayer A, Cosgrove SE, et al. Clinical practice guidelines by the infectious diseases society of america for the treatment of methicillin-resistant Staphylococcus aureus infections in adults and children. Clin Infect Dis. 2011;52:e18-55.

16. Deresinski S. Vancomycin: does it still have a role as an antistaphylococcal agent? Expert Rev Anti Infect Ther. 2007;5:393-401.

17. Deresinski S. Counterpoint: vancomycin and Staphylococcus aureus-an antibiotic enters obsolescence. Clin Infect Dis. 2007;44:1543-8.

18. Rybak M, Lomaestro B, Rotschafer JC, et al. Therapeutic monitoring of vancomycin in adult patients: a consensus review of the American Society of Health-System Pharmacists, the Infectious Diseases Society of America, and the Society of Infectious Diseases Pharmacists. Am J Health Syst Pharm. 2009;66:82-98.

19. van Hal SJ, Paterson DL, Lodise TP. Systematic review and meta-analysis of vancomycin-induced nephrotoxicity associated with dosing schedules that maintain troughs between 15 and $20 \mathrm{mg}$ per liter. Antimicrob Agents Chemother. 2013;57: $734-44$.

20. Bosso JA, Nappi J, Rudisill C, et al. Relationship between vancomycin trough concentrations and nephrotoxicity: a prospective multicenter trial. Antimicrob Agents Chemother. 2011;55:5475-9.

21. O'Riordan W, Green S, Overcash JS, et al. Omadacycline for acute bacterial skin and skin-structure infections. N Engl J Med. 2019;380:528-38.

22. Pullman J, Gardovskis J, Farley B, et al. Efficacy and safety of delafloxacin compared with vancomycin plus aztreonam for acute bacterial skin and skin structure infections: a Phase 3, double-blind, randomized study. J Antimicrob Chemother. 2017;72: 3471-80.

23. Corey GR, Wilcox M, Talbot GH, et al. Integrated analysis of CANVAS 1 and 2: phase 3, multicenter, randomized, double-blind studies to evaluate the safety and efficacy of ceftaroline versus vancomycin plus aztreonam in complicated skin and skin-structure infection. Clin Infect Dis. 2010;51:641-50.

24. Breedt J, Teras J, Gardovskis J, et al. Safety and efficacy of tigecycline in treatment of skin and skin structure infections: results of a double-blind phase 3 comparison study with vancomycin-aztreonam. Antimicrob Agents Chemother. 2005;49:4658-66.

25. Kamath RS, Sudhakar D, Gardner JG, et al. Guidelines vs actual management of skin and soft tissue infections in the emergency department. Open Forum Infect Dis. 2018;5:188. 
26. Mueller K, McCammon C, Skrupky L, et al. Vancomycin use in patients discharged from the emergency department: a retrospective observational cohort study. J Emerg Med. 2015;49:50-7.

27. Minejima E, Choi J, Beringer P, et al. Applying new diagnostic criteria for acute kidney injury to facilitate early identification of nephrotoxicity in vancomycin-treated patients. Antimicrob Agents Chemother. 2011;55:3278-83.

28. Cano EL, Haque NZ, Welch VL, et al. Incidence of nephrotoxicity and association with vancomycin use in intensive care unit patients with pneumonia: retrospective analysis of the IMPACT-HAP database. Clin Ther. 2012;34:149-57.

29. Kullar R, Davis SL, Levine DP, et al. Impact of vancomycin exposure on outcomes in patients with methicillin-resistant Staphylococcus aureus bacteremia: support for consensus guidelines suggested targets. Clin Infect Dis. 2011;52:975-81.

30. Finch NA, Zasowski EJ, Murray KP, et al. A Quasiexperiment to study the impact of vancomycin area under the concentration-time curve-guided dosing on vancomycin-associated nephrotoxicity. Antimicrob Agents Chemother. 2017;61:e01293.

31. Neely MN, Kato L, Youn G, et al. Prospective trial on the use of trough concentration versus area under the curve to determine therapeutic vancomycin dosing. Antimicrob Agents Chemother. 2018;62:e02042.

32. Shields RK, Nguyen MH, Chen L, et al. Ceftazidimeavibactam is superior to other treatment regimens against carbapenem-resistant Klebsiella pneumoniae bacteremia. Antimicrob Agents Chemother. 2017;61:e00883.

33. Wong-Beringer A, Joo J, Tse E, et al. Vancomycinassociated nephrotoxicity: a critical appraisal of risk with high-dose therapy. Int J Antimicrob Agents. 2011;37:95-101.

34. Choi YC, Saw S, Soliman D, et al. Intravenous vancomycin associated with the development of nephrotoxicity in patients with class III obesity. Ann Pharmacother. 2017;51:937-44.

35. Navalkele B, Pogue JM, Karino S, et al. Risk of acute kidney injury in patients on concomitant vancomycin and piperacillin-tazobactam compared to those on vancomycin and cefepime. Clin Infect Dis. 2017;64:116-23.

36. Cockcroft DW, Gault MH. Prediction of creatinine clearance from serum creatinine. Nephron. 1976;16:31-41.

37. Levy MM, Fink MP, Marshall JC, et al. 2001 SCCM/ ESICM/ACCP/ATS/SIS international sepsis definitions conference. Intensive Care Med. 2003;29:530-8.

38. Khwaja A. KDIGO clinical practice guidelines for acute kidney injury. Nephron Clin Pract. 2012;120: c179-84.

39. Harris PA, Taylor R, Thielke R, et al. Research electronic data capture (REDCap) - a metadata-driven methodology and workflow process for providing translational research informatics support. J Biomed Inform. 2009;42:377-81.

40. Charlson ME, Pompei P, Ales KL, et al. A new method of classifying prognostic comorbidity in longitudinal studies: development and validation. J Chronic Dis. 1987;40:373-83.

41. Cutfield T, Walter H, Hobbs M, et al. Association of the Dundee severity classification with mortality, length of stay and readmission in adult inpatients with cellulitis. J Antimicrob Chemother. 2019;74: 200-6.

42. Seymour CW, Liu VX, Iwashyna TJ, et al. Assessment of clinical criteria for sepsis: for the third international consensus definitions for sepsis and septic shock (sepsis-3). JAMA. 2016;315:762-74.

43. Marwick C, Broomhall J, McCowan C, et al. Severity assessment of skin and soft tissue infections: cohort study of management and outcomes for hospitalized patients. J Antimicrob Chemother. 2011;66: 387-97.

44. Levey AS, Bosch JP, Lewis JB, et al. A more accurate method to estimate glomerular filtration rate from serum creatinine: a new prediction equation. Modification of Diet in Renal Disease Study Group. Ann Intern Med. 1999;130:461-70.

45. Levey AS, Stevens LA, Schmid CH, et al. A new equation to estimate glomerular filtration rate. Ann Intern Med. 2009;150:604-12.

46. Bellomo R, Ronco C, Kellum JA, et al. Acute renal failure-definition, outcome measures, animal models, fluid therapy and information technology needs: the Second International Consensus Conference of the Acute Dialysis Quality Initiative (ADQI) Group. Crit Care. 2004;8:R204-12.

47. Mehta RL, Kellum JA, Shah SV, et al. Acute kidney injury network: report of an initiative to improve outcomes in acute kidney injury. Crit Care. 2007;11:R31.

48. Lodise TP, Patel N, Lomaestro BM, et al. Relationship between initial vancomycin concentrationtime profile and nephrotoxicity among hospitalized patients. Clin Infect Dis. 2009;49:507-14. 
49. Magill SS, Edwards JR, Beldavs ZG, et al. Prevalence of antimicrobial use in US acute care hospitals, May-September 2011. JAMA. 2014;312:1438-46.

50. Sinha Ray A, Haikal A, Hammoud KA, et al. Vancomycin and the risk of AKI: a systematic review and meta-analysis. Clin J Am Soc Nephrol. 2016;11: 2132-40.

51. Pfunter A, Wier LM, Steiner C. Costs for hospital stays in the United States, 2010. Healthcare cost and utilization project statistical brief. Agency for healthcare Quality and Research. https://hcup-us. ahrq.gov/reports/statbriefs/sb146.pdf Accessed 08 June 2019.

52. Lodise TP, Lomaestro B, Graves J, et al. Larger vancomycin doses (at least four grams per day) are associated with an increased incidence of nephrotoxicity. Antimicrob Agents Chemother. 2008;52: 1330-6.

53. Jeffres $\mathrm{MN}$, Isakow $\mathrm{W}$, Doherty JA, et al. A retrospective analysis of possible renal toxicity associated with vancomycin in patients with health careassociated methicillin-resistant Staphylococcus aureus pneumonia. Clin Ther. 2007;29:1107-15.

54. Royer S, DeMerle KM, Dickson RP, et al. Shorter versus longer courses of antibiotics for infection in hospitalized patients: a systematic review and metaanalysis. J Hosp Med. 2018;13:336-42.

55. Spellberg B. The maturing antibiotic mantra: "Shorter is still better". J Hosp Med. 2018;13(361):2.

56. Beben T, Rifkin DE. GFR estimating equations and liver disease. Adv Chronic Kidney Dis. 2015;22: 337-42.

57. Eells SJ, Chira S, David CG, et al. Non-suppurative cellulitis: risk factors and its association with Staphylococcus aureus colonization in an area of endemic community-associated methicillin-resistant $S$. aureus infections. Epidemiol Infect. 2011;139: 606-12.
58. Jeng A, Beheshti M, Li J, et al. The role of betahemolytic streptococci in causing diffuse, nonculturable cellulitis: a prospective investigation. Medicine (Baltimore). 2010;89:217-26.

59. Alexopoulos E. Drug-induced acute interstitial nephritis. Ren Fail. 1998;20:809-19.

60. Jorgensen SCJ, Mercuro NJ, Davis SL, et al. Delafloxacin: place in therapy and review of microbiologic, clinical and pharmacologic properties. Infect Dis Ther. 2018;7:197-217.

61. Rybak JM, Roberts K. Tedizolid Phosphate: a nextgeneration oxazolidinone. Infect Dis Ther. 2015;4: $1-4$.

62. Miller LG, Daum RS, Creech CB, et al. Clindamycin versus trimethoprim-sulfamethoxazole for uncomplicated skin infections. N Engl J Med. 2015;372: 1093-103.

63. Moran GJ, Krishnadasan A, Mower WR, et al. Effect of cephalexin plus trimethoprim-sulfamethoxazole vs cephalexin alone on clinical cure of uncomplicated cellulitis: a randomized clinical trial. JAMA. 2017;317:2088-96.

64. Bassetti M, Baguneid M, Bouza E, et al. European perspective and update on the management of complicated skin and soft tissue infections due to methicillin-resistant Staphylococcus aureus after more than 10 years of experience with linezolid. Clin Microbiol Infect. 2014;20(Suppl 4):3-18.

65. Barlam TF, Cosgrove SE, Abbo LM, et al. Implementing an antibiotic stewardship program: guidelines by the infectious diseases society of america and the society for healthcare epidemiology of America. Clin Infect Dis. 2016;62:e51-77.

66. Luther MK, Timbrook TT, Caffrey AR, et al. Vancomycin plus piperacillin-tazobactam and acute kidney injury in adults: a systematic review and meta-analysis. Crit Care Med. 2018;46:12-20. 